\title{
Topological Dynamics in Tandem with Permutation Groups
}

\author{
Isaac Kwame Dontwi, William Obeng-Denteh, Stephen K. Manu, Richard Nyarko Yeboah \\ Department of Mathematics, College of Science, Kwame Nkrumah University of Science and Technology, Kumasi, Ghana \\ Email: ikedontwi@hotmail.com,obengdentehw@yahoo.com,deoxy2005@yahoo.com, aricky2008@yahoo.com
}

Received September 14, 2012; revised November 16, 2012; accepted December 3, 2012

\begin{abstract}
The purpose of this study was to delve into the aspects of abstract algebra that has a link with topological dynamics in terms of permutation and symmetric groups. This would aid users to appreciate the role it plays in the theory and application of topological dynamics. The usage of matlab programming to carry out the permutations was carried out. The study contributes to the literature by providing candid explanation and usage of data-based evidence documenting the extent to which topological dynamics operates.
\end{abstract}

Keywords: Permutation Groups; Recurrence; Topological Dynamics; Matlab Code; Flows

\section{Introduction}

Topological dynamics in [1] and [2] form an aspect of the theory of dynamical systems in [3] where qualitative [4], asymptotic [5] properties [6-9] of dynamical systems are taken into account from the viewpoint of general topology in [10]. Reference [2] provides the first self-contained comprehensive exposition of the theory of dynamical systems as a core mathematical discipline closely intertwined with most of the main areas of mathematics. Topological dynamics is defined as the study of asymptotic [11-14] or long term properties of families of maps in [15] of topological spaces. Abstract topological dynamics is usually developed in the context of flows as in [16]. A flow $(X, T)$ is a jointly continuous action as in [17] of the topological group [18] $T$ on the topological space $X$ [19]. This means that there is a continuous map [20] from $X \times T \rightarrow X,(t, x) \mapsto t x$ with $e x=x$ and $t(x s)=(t s) x$, which clearly indicates that $\mathrm{e}$ is the identity of $T, t, s \in T$ and $\in X$. A topological dynamical system is the central point in the study of topological dynamics. This constitutes a topological space, in tandem with a continuous transformation, a continuous flow, in other words a semigroup of continuous transformations of that space. This state of affairs brings the activity of permutation groups [21-23] and semigroups action [24] into the scenario.

Recorded studies of permutations of the earliest periods can be found in Sefer Yetsirah or Book of Creation which was written by an unknown Jewish author sometime before the eighth century [25]. This was improved upon with the passage of time.

\section{Problem Statement and the Way Forward}

The main problem that was considered was to identify the link between topological dynamics and permutation groups. The usage of permutation groups was used synonymously with Cartesian product. It became necessary to decipher the link based on the underlying factors enumerated stemming from the laid down rules from credible sources [26]. A periodic point returns to itself every hour on the hour; but almost periodic point returns to a neighbourhood every hour within the hour. This is what has been established to lend credence to topological dynamics in tandem with permutation groups.

\section{Preliminary Notations and Definitions}

A topological transformation group, or transformation group is explained to be an ordered triple $(X, T, \pi)$ such that the following conditions are satisfied:

1) $X$ is a topological space which is called the phase space: $T$ is called the topological group known as the phase space and $\pi$ is a map of the cartesian product $X \times T$ into $X$, called the phase map;

2) $x e=x$ for all $x \in X$ where e denotes the identity element of $T$, and the value of $\pi$ at the point $(x, t)$ of $X \times T$ be represented by $x t$;

3) $\pi$ is continuous.

In analyzing a transformation group $(X, T, \pi)$ the phase map $(x, t) \rightarrow x t$ gives the values of two kinds of 
maps when one of the variables $x, t$ is replaced by a constant. They are transition and motion in [27]. Depending on additive groups of real numbers or integers a continuous flow or discrete flow arises. These two kinds of flows are related in that a continuous flow determines many discrete flows by taking cyclic subgroups of $R$. Conversely, a discrete flow determines a continuous flow when the phase space $X$ is extended to the Cartesian product of $X$ and the closed interval.

The crux of the work here cruises on recurrence. Suppose $\varnothing$ is a homeomorphism of $X$ onto $X$, a point $x$ of the phase space is said be almost periodic under $\varnothing$ and $\varnothing$ is said to be almost periodic at $x$ provided that if $U$ is a neighbourhood of $x$, then there exists a relatively dense subset $A$ of additive group of integers such that $x \varnothing^{n} \in U$ for all $n \in A$. A periodic point returns to itself every hour on the hour; but almost periodic point returns to a neighbourhood every hour within the hour. This is what has been established to lend credence to topological dynamics in tandem with permutation groups.

\section{Methods}

A permutation group is a group of permutations on a finite set of positive integers. Permutation groups are therefore all operations for groups which can be applied to them. Here permutation multiplications are carried out on permutations of the main set. $S_{3}$ is seen as a group $D_{3}$ of symmetries of an equilateral triangle in which two copies of it with vertices 1,2 , and 3 can be placed with one covering the other with vertices on top of vertices in [28]. The subscripted Greek letters $\rho_{i}$ and $\mu_{i}$ are for rotations and mirror images in bisectors of angles. The second one is the $D_{4}$ which is the group of symmetries of the square. The subscripted Greek letters $\rho_{i}, \mu_{i}$ and $\delta_{i}$ are for rotations, mirror images in perpendiculars bisectors of sides, and for diagonal flips respectively. Matlab was then used to develop codes for the permutations.

\section{Results and Discussion}

Two important examples would be explored to unearth the surprising revelations in the processes. See Figure 1 for the operations of the matlab code. Considering the group $S_{3}$ with $3 !=6$ elements where $A=\{1,2,3\}$. The permutations are listed with names using Greek letters assigned to them viz.;

$$
\begin{aligned}
& \rho_{0}=\left(\begin{array}{lll}
1 & 2 & 3 \\
1 & 2 & 3
\end{array}\right), \rho_{1}=\left(\begin{array}{lll}
1 & 2 & 3 \\
2 & 3 & 1
\end{array}\right), \rho_{2}=\left(\begin{array}{lll}
1 & 2 & 3 \\
3 & 1 & 2
\end{array}\right) \\
& \mu_{1}=\left(\begin{array}{lll}
1 & 2 & 3 \\
1 & 3 & 2
\end{array}\right), \mu_{2}=\left(\begin{array}{lll}
1 & 2 & 3 \\
3 & 2 & 1
\end{array}\right), \mu_{3}=\left(\begin{array}{lll}
1 & 2 & 3 \\
2 & 1 & 3
\end{array}\right)
\end{aligned}
$$

Now,

$$
\begin{aligned}
& \rho_{0} \rho_{0}=\left(\begin{array}{lll}
1 & 2 & 3 \\
1 & 2 & 3
\end{array}\right)\left(\begin{array}{lll}
1 & 2 & 3 \\
1 & 2 & 3
\end{array}\right)=\left(\begin{array}{lll}
1 & 2 & 3 \\
1 & 2 & 3
\end{array}\right)=\rho_{0} \\
& \rho_{0} \rho_{1}=\left(\begin{array}{lll}
1 & 2 & 3 \\
1 & 2 & 3
\end{array}\right)\left(\begin{array}{lll}
1 & 2 & 3 \\
2 & 3 & 1
\end{array}\right)=\left(\begin{array}{lll}
1 & 2 & 3 \\
2 & 3 & 1
\end{array}\right)=\rho_{1} \\
& \rho_{0} \rho_{2}=\left(\begin{array}{lll}
1 & 2 & 3 \\
1 & 2 & 3
\end{array}\right)\left(\begin{array}{lll}
1 & 2 & 3 \\
3 & 1 & 2
\end{array}\right)=\left(\begin{array}{lll}
1 & 2 & 3 \\
3 & 1 & 2
\end{array}\right)=\rho_{2}
\end{aligned}
$$

The detailed process of the permutations can be found in [28].

The multiplication table for $S_{3}$ is depicted in Table 1.

The next is to form the dihedral-group $D_{4}$ of permutations to the ways that two copies of a square with vertices 1-4 can be placed with one covering the other with vertices on top of vertices. $D_{4}$ is the group of symmetries of the square.

The following representations are used;

$$
\begin{aligned}
& \rho_{0}=\left(\begin{array}{llll}
1 & 2 & 3 & 4 \\
1 & 2 & 3 & 4
\end{array}\right), \rho_{1}=\left(\begin{array}{llll}
1 & 2 & 3 & 4 \\
2 & 3 & 4 & 1
\end{array}\right), \\
& \rho_{2}=\left(\begin{array}{llll}
1 & 2 & 3 & 4 \\
3 & 4 & 1 & 2
\end{array}\right), \rho_{3}=\left(\begin{array}{llll}
1 & 2 & 3 & 4 \\
4 & 1 & 2 & 3
\end{array}\right) \\
& \mu_{1}=\left(\begin{array}{llll}
1 & 2 & 3 & 4 \\
2 & 1 & 4 & 3
\end{array}\right), \mu_{2}=\left(\begin{array}{llll}
1 & 2 & 3 & 4 \\
4 & 3 & 2 & 1
\end{array}\right) \text {, } \\
& \delta_{1}=\left(\begin{array}{llll}
1 & 2 & 3 & 4 \\
3 & 2 & 1 & 4
\end{array}\right), \delta_{2}=\left(\begin{array}{llll}
1 & 2 & 3 & 4 \\
1 & 4 & 3 & 2
\end{array}\right)
\end{aligned}
$$

Now

$$
\begin{aligned}
\rho_{0} \rho_{0} & =\left(\begin{array}{llll}
1 & 2 & 3 & 4 \\
1 & 2 & 3 & 4
\end{array}\right)\left(\begin{array}{llll}
1 & 2 & 3 & 4 \\
1 & 2 & 3 & 4
\end{array}\right) \\
& =\left(\begin{array}{llll}
1 & 2 & 3 & 4 \\
1 & 2 & 3 & 4
\end{array}\right)=\rho_{0} \\
\rho_{0} \rho_{1} & =\left(\begin{array}{llll}
1 & 2 & 3 & 4 \\
1 & 2 & 3 & 4
\end{array}\right)\left(\begin{array}{llll}
1 & 2 & 3 & 4 \\
2 & 3 & 4 & 1
\end{array}\right) \\
& =\left(\begin{array}{llll}
1 & 2 & 3 & 4 \\
2 & 3 & 4 & 1
\end{array}\right)=\rho_{1} \\
\rho_{0} \rho_{2} & =\left(\begin{array}{llll}
1 & 2 & 3 & 4 \\
1 & 2 & 3 & 4
\end{array}\right)\left(\begin{array}{llll}
1 & 2 & 3 & 4 \\
3 & 4 & 1 & 2
\end{array}\right) \\
& =\left(\begin{array}{llll}
1 & 2 & 3 & 4 \\
3 & 4 & 1 & 2
\end{array}\right)=\rho_{2}
\end{aligned}
$$

The multiplication table for $S_{4}$ is shown in Table 2.

Theorem: (Cayley's Theorem) every group is isormorphic to a group of permutations. An important addition is that in group theory, Cayley's theorem which was 


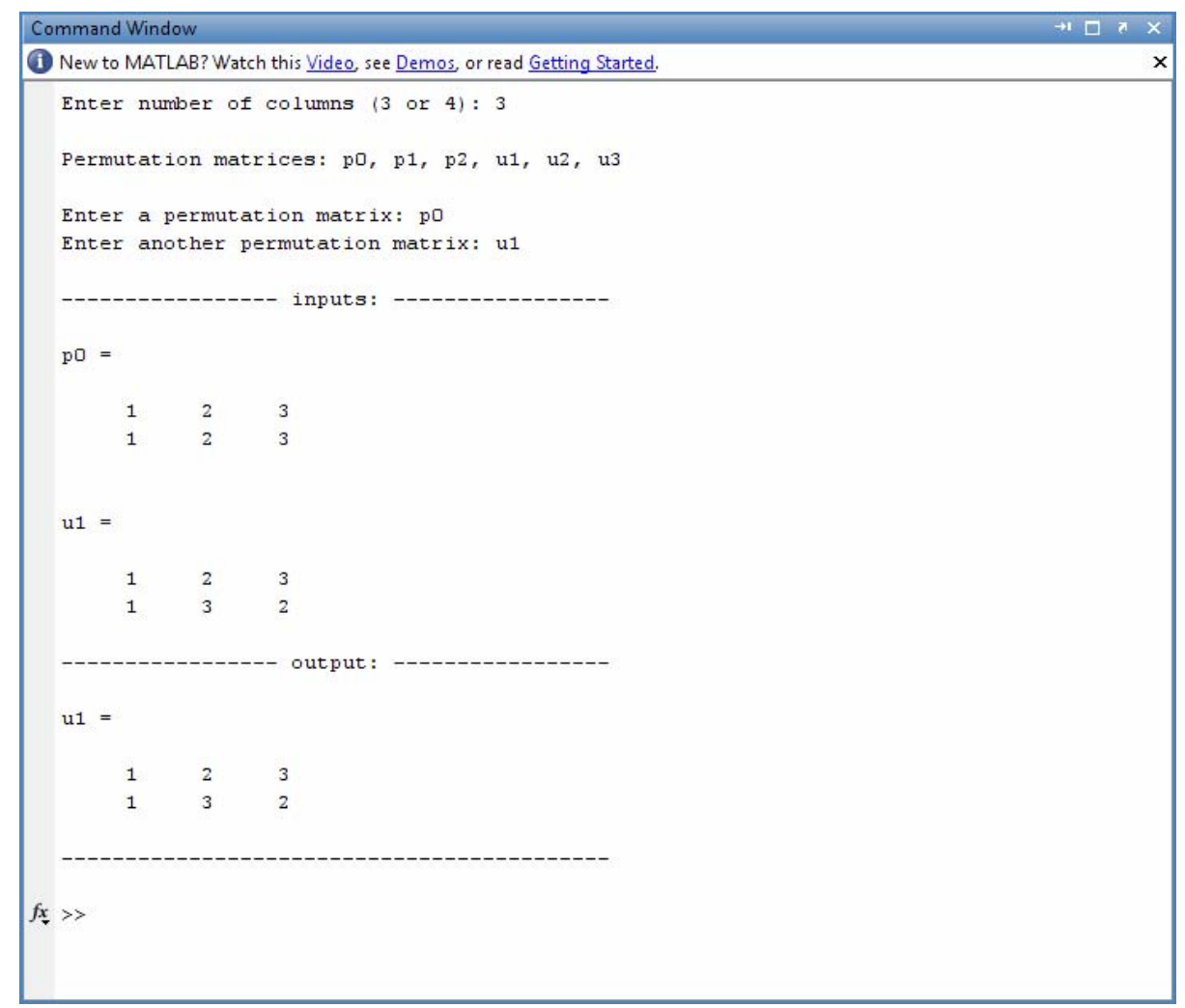

Figure 1. Pou1 has been depicted.

Table 1. The multiplication table for $S_{3}$.

\begin{tabular}{ccccccc}
\hline & $\rho_{0}$ & $\rho_{1}$ & $\rho_{2}$ & $\mu_{1}$ & $\mu_{2}$ & $\mu_{3}$ \\
\hline$\rho_{0}$ & $\rho_{0}$ & $\rho_{1}$ & $\rho_{2}$ & $\mu_{1}$ & $\mu_{2}$ & $\mu_{3}$ \\
$\rho_{1}$ & $\rho_{1}$ & $\rho_{2}$ & $\rho_{0}$ & $\mu_{3}$ & $\mu_{1}$ & $\mu_{2}$ \\
$\rho_{2}$ & $\rho_{2}$ & $\rho_{0}$ & $\rho_{1}$ & $\mu_{2}$ & $\mu_{3}$ & $\mu_{1}$ \\
$\mu_{1}$ & $\mu_{1}$ & $\mu_{2}$ & $\mu_{3}$ & $\rho_{0}$ & $\rho_{1}$ & $\rho_{2}$ \\
$\mu_{2}$ & $\mu_{2}$ & $\mu_{3}$ & $\mu_{1}$ & $\rho_{2}$ & $\rho_{0}$ & $\rho_{1}$ \\
$\mu_{3}$ & $\mu_{3}$ & $\mu_{1}$ & $\mu_{2}$ & $\rho_{1}$ & $\rho_{2}$ & $\rho_{0}$ \\
\hline
\end{tabular}

Table 2. The multiplication table for $S_{4}$.

\begin{tabular}{ccccccccc}
\hline & $\rho_{0}$ & $\rho_{1}$ & $\rho_{2}$ & $\rho_{3}$ & $\mu_{1}$ & $\mu_{2}$ & $\delta_{1}$ & $\delta_{2}$ \\
\hline$\rho_{0}$ & $\rho_{0}$ & $\rho_{1}$ & $\rho_{2}$ & $\rho_{3}$ & $\mu_{1}$ & $\mu_{2}$ & $\delta_{1}$ & $\delta_{2}$ \\
$\rho_{1}$ & $\rho_{1}$ & $\rho_{2}$ & $\rho_{3}$ & $\rho_{0}$ & $\delta_{1}$ & $\delta_{2}$ & $\mu_{2}$ & $\mu_{1}$ \\
$\rho_{2}$ & $\rho_{2}$ & $\rho_{3}$ & $\rho_{0}$ & $\rho_{1}$ & $\mu_{2}$ & $\mu_{1}$ & $\delta_{2}$ & $\delta_{1}$ \\
$\rho_{3}$ & $\rho_{3}$ & $\rho_{0}$ & $\rho_{1}$ & $\rho_{2}$ & $\delta_{2}$ & $\delta_{1}$ & $\mu_{1}$ & $\mu_{2}$ \\
$\mu_{1}$ & $\mu_{1}$ & $\delta_{2}$ & $\mu_{2}$ & $\delta_{1}$ & $\rho_{0}$ & $\rho_{2}$ & $\rho_{3}$ & $\rho_{1}$ \\
$\mu_{2}$ & $\mu_{2}$ & $\delta_{1}$ & $\mu_{1}$ & $\delta_{2}$ & $\rho_{2}$ & $\rho_{0}$ & $\rho_{1}$ & $\rho_{3}$ \\
$\delta_{1}$ & $\delta_{1}$ & $\mu_{1}$ & $\delta_{2}$ & $\mu_{2}$ & $\rho_{1}$ & $\rho_{3}$ & $\rho_{0}$ & $\rho_{2}$ \\
$\delta_{2}$ & $\delta_{2}$ & $\mu_{2}$ & $\delta_{1}$ & $\mu_{1}$ & $\rho_{3}$ & $\rho_{1}$ & $\rho_{2}$ & $\rho_{0}$ \\
\hline
\end{tabular}


named in honour of Arthur Cayley, states that every group $G$ is isomorphic to a subgroup of the symmetric group acting on $G$ in [28]. In fact this can be explained as an instance of the group action of $G$ on the elements of $G$. A permutation of a set $G$ is any objective function taking $G$ onto $G$; and the set of all such functions forms a group under function composition, called the symmetric group on $G$, and this denoted by $\operatorname{Sym}(G)$.

Cayley's theorem outlines all groups on the same pedestal, by taking into account any group which might be made up of infinite groups such as $(\boldsymbol{R},+)$ as a permutation group of some underlying set. This establishes the fact that theorems which holds for permutation groups are counted to hold for groups in general.

The group being used here satisfies the axioms for topological group. In topological dynamics it involves topological groups and their operations [2,27].

\section{Using Matlab to Compute the Results above Instead of Manual Approach}

\section{Algorithm}

In general this work has been trying to establish a link between topological dynamics and permutation groups which has been likened to Cartesian product. Matlab has been used to generate the permutations explained above. See Figure 1 for an example of the usage of the matlab code. The code could be extended to cover $S_{n}$.

\section{Conclusions}

The crux of the work here cruised on recurrence in [29]. A periodic point returns to itself every hour on the hour; but almost periodic point returns to a neighbourhood every hour within the hour. The recurrence was depicted in the permutations that were done. All the parameters in the form of the Greek symbols recurred in the table provided. When this trend is continued for all time the recurrence nature would be mimicked along the same trend. This is what has been established to lend credence to topological dynamics in tandem with permutation groups.

It is worthy to note that from all the tables each column gives a permutation of the group set. It is obvious that at least every finite group is isomorphic to a subgroup of the group. Isomorphism is about one-to-one correspondence satisfying a particular relation. This is the way topological dynamics apply permutation in finding the symmetries of objects and their rotations as well and matlab has been useful over here.

\section{REFERENCES}

[1] E. Glasner and B. Weiss, "On the Interplay between Measurable and Topological Dynamics," Most, Vol. 1, 2004, pp. 1-47.
[2] A. Katok and B. Hasselblatt, "Introduction to the Modern Theory of Dynamical Systems," October, Cambridge University Press, Cambridge, Vol. 54, 1995, pp. 519-522. doi:10.1017/CBO9780511809187.018

[3] J. P. Boon and O. Decroly, "Dynamical Systems Theory for Music Dynamics," Chaos, Vol. 5, No. 3, 1994, pp. 501-508. doi:10.1063/1.166145

[4] E. Ball, "Qualitative Research," Palgrave Macmillan, London, Vol. 5, 2011, pp. 285-306.

[5] N. Kruse, "Global Asymptotic Stability in Some Discrete Dynamical Systems," Journal of Mathematical Analysis and Applications, Vol. 235, No. 1, 1999, pp. 151-158. doi:10.1006/jmaa.1999.6384

[6] N. Chernov, "On Some Statistical Properties of Dynamical Systems," Proceedings of the 4th Berkeley Symposium on Mathematical Statistics and Probability, Vol. 3, 2008, pp. 315-320.

[7] R. Markarian, "Statistical Properties of Dynamical Systems with Singularities," Journal of Statistical Physics, Vol. 80, No. 5-6, 1995, pp. 1207-1239. doi:10.1007/BF02179869

[8] W. Mitkowski, "Scaling Properties of Discrete Dynamical Systems," Bulletin of the Polish Academy of Sciences Technical Sciences, Vol. 56, No. 4, 2008.

[9] R. A. Johnson, K. J. Palmerand and G. R. Sell, "Ergodic Properties of Linear Dynamical Systems," SIAM Journal on Mathematical Analysis, Vol. 18, No. 2, 1987, pp. 191202. doi:10.1137/0518001

[10] J. M. Møller, “General Topology,” Topology, 2009, pp. 1-70.

[11] B. Audoly, "Asymptotic Study of the Interfacial Crack with Friction," Journal of the Mechanics and Physics of Solids, Vol. 48, No. 9, 2000, pp. 1851-1864. doi:10.1016/S0022-5096(99)00098-8

[12] K. Hellan, "An Asymptotic Study of Slow Radial Cracking," International Journal of Fracture, Vol. 26, No. 1, 1984, pp. 17-30. doi:10.1007/BF01152309

[13] C. Song, H. Gao and X. W. Zheng, "A Study of Asymptotic Stability for Delayed Recurrent Neural Networks," IEEE International Symposium on Circuits and Systems, Taipei, 24-27 May 2009, pp. 2125-2128.

[14] L. Beiraodaveiga, "Asymptotic Study of the Solution for Pinched Cylindrical Shells," Computer Methods in Applied Mechanics and Engineering, Vol. 194, No. 9-11, 2005, pp. 1113-1139. doi:10.1016/j.cma.2004.06.015

[15] J. L. Kelley, "General Topology," Van Nostrand, Princeton, 1955.

[16] C. Othmer, "A Continuous Adjoint Formulation for the Computation of Topological and Surface Sensitivities of Ducted Flows," Topology, Vol. 58, 2008, pp. 861-877.

[17] D. G. Davis, "Continuous Action," Journal of Pure and Applied Algebra, Vol. 206, 2006, pp. 322-354. doi:10.1016/i.jpaa.2005.06.022

[18] D. Repovs and L. Zdomskyy, "A New Lindelof Topological Group," Topology and Its Applications, Vol. 157, No. 5, 2010, pp. 1-8.

[19] O. Pavlov, "A First Countable Linearly Lindelöf Not 
Lindelöf Topological Space," Topology and Its Applications, Vol. 1, 2005, pp. 1-9.

[20] C. Adams and R. Franzoza, "Introduction to Topology: Pure and Applied," Pearson Prentice Hall, 2008.

[21] P. J. Cameron, "Permutation Groups," In R. L. Graham, M. Grotschel and L. Lovasz, Eds., Handbook of Combinatorics, Elsevier and the MIT Press, Vol. 1, 1995.

[22] S. Shelah and S. Thomas, "Homogeneity of Infinite Permutation Groups," Archive for Mathematical Logic, Vol. 28, No. 2, 1989, pp. 143-147. doi:10.1007/BF01633987

[23] J. Bichon, "Algebraic Quantum Permutation Groups," Asian European Journal of Mathematics, 2006. http://Arxiv.Org/Abs/0710.1521

[24] J. Renshaw, "Inverse Semigroups Acting On Graphs," World Scientific, 2010.

http://www.worldscibooks.com/Mathematics/5584.html
[25] V. Katz, "Historical Notes," In: J. B. Fraleigh, A First Course in Abstract Algebra, Pearson Education Inc., Boston, 2003.

[26] G. R. Sell, “Topological Dynamics and Ordinary Differential Equations," Van Nostrand-Reinhold Company, London, 1971.

[27] W. H. Gottschalk, "Minimal Sets: An Introduction to Topological Dynamics," Bulletin of the American Mathematical Society, Vol. 64, 1958, pp. 336-351. doi:10.1090/S0002-9904-1958-10223-2

[28] J. B. Fraleigh, "A First Course in Abstract Algebra," Pearson Education Inc., Boston, 2003.

[29] W. Obeng-Denteh, "On the Study of Topological Dynamical Systems," PhD Thesis Draft, Department of Mathematics, Kwame Nkrumah University of Science and Technology, Kumasi, 2012. 